\title{
Case Report Percutaneous Dilational Tracheostomy A bed side procedure in the intensive care unit
}

Dr Goitom Hagos( MD), Dr Dania Serrano(MD)

Orotta Medical Surgical National Referal Hospital

\section{Introduction:}

Tracheostomy is one of the most commonly performed procedures in critically ill patients. Percutaneous dilatational tracheostomy (PDT), according to ciaglias technique described in 1985, has become the most popular technique for percutaneous tracheostomy and is demonstrably as safe as surgical tracheostomy. In 1999, an extensively modified technique of PDT was introduced, the Ciaglia Blue Rhino that consists of one step dilatation by means of curved dilator with hydrophilic coating. Tracheostomy is performed commonly to aid weaning from assisted ventilation in patients who require longer intensive care and assisted ventilation.

We report the use of a bed side percutaneous tracheaostomy technique in adult patient who required intensive care and longer assisted ventilation after drowning.

\section{CASE REPORT}

A 60 year old woman was admitted to our intensive care unit Orotta medical surgical national referral Hospital after having been found immersed in a water. Patient was in Adi Rraessi for holy water blessed by a priest, which is $12 \mathrm{Km}$ away from the capital. The exact period of immersion was not known. After being rescued from the water she was found to be apnoeic and cyanosed. Attempts were made to resuscitate patient and as she started to breath with difficult she was transferred immediately to the hospital.

On arrival to the Emergency department she was gasping, cyanosed and unconscious. Patient was given oxygen by face mask and frequent suction performed.. Despite all measures given in the emergency department patient remained critical and was transferred to ICU on 16-6-08.

On arrival to our ICU patient was gasping, cyanosed, and unconscious. Her pulse rate was 146 beats per minute; blood pressure was $130 / 70 \mathrm{mmHg}$ and temperature was $36^{\circ} \mathrm{C} R$; $56 /$ minute and her oxygen saturation was $45 \%$. Patient was in severe respiratory distress with flering of ali nasi, intercostals and sub costal retractions. Her pupils were small and reactive. There was full of inspiratory and expiratory crepitations over both lung fields. Cardiac examinations revealed only tachycardia. Abdominal examination revealed no abnormality and had no leg oedema.

\section{Investigation:}

Her $\mathrm{Hb}$ was $11 \mathrm{gm} / \mathrm{dl}$ WBC $14,7000 / \mathrm{mm} 3$ platelets 230,000 , urinalysis, electrolytes, blood sugar, renal and liver function tests were essentially normal. ECG showed sinus tachycardia with signs of low voltage and LBBB. Urgent chest radiograph taken after intubation demonstrated diffuse, extensive bilateral interstitial and alveolar infiltrates with signs of pulmonary congestion. Blood gas analysis could not be determined on the patient

Patient was intubated immediately and intermittent positive pressure ventilation was commenced using Bennt ventilator. Positive End Expiratory pressure (PEEP) was applied and Patient was closely monitored. Monitoring consisted of, blood pressure, oxygen saturation by pulse oximetry temperature and hourly urine out put. In addition she was put on continuous cardiac monitor.

Frequent tracheal suction performed yielded copious frothy secretions and a wide bore nasogastric tube was also inserted.

Though the patient was hemodynamically stable her oxygen saturation remained unsatisfactory despite high inspired oxygen concentration. Positive end expiratory pressure was adjusted in attempt to improve oxygenation at $12.5 \mathrm{~cm}$ water. Patient remained stable on ventilatory support and the standard conventional treatment for her ARDS continued.. Her oxygen saturation remained stable and maintained above $90 \%$. Regardless of the management to her ARDS was difficult to wean patient from the ventilator. Subsequent clinical evaluation revealed no significant improvement of her chest findings. Control chest radiography done two weeks later showed no marked radiological improvement. Attempts made to wean her from mechanical ventilation were not successful. Three weeks later PDT was planned, because of repeated inability to wean the patient from ventilatory support. After obtaining informed consent from families we performed a bed side ICU procedure on the patient.

Patient was positioned with an appropriate degree of neck extension in the tracheostomy position, and the site was infiltrated with $10 \mathrm{ml}$ of lidocane $1 \%$.Flexible bronchoscopy was not used during this procedure. Under direct vision, the oral tracheal tube was deflated and withdrawn approximately $1 \mathrm{~cm}$. An experienced anaesthetist maintained this position .Ultrasound guide and support was also given by our radiologist.

A catheter introducer needle is inserted at the mid line between the second and third tracheal rings. Entrance in to the tracheal lumen confirmed by aspiration of free air bubbles into a saline filled syringe. A guide wire was then inserted through the cannula, and the cannula was removed. A small dilator, supplied with PT Kit, was then placed over the wire guide in to the trachea. An air leak confirmed correct placement. The trachea was then dialated to an appropriate size with the specialized, Ciaglia Blue Rhino dilator. A tracheostomy tube was then introduced in to the 
trachea. Frequent suction performed to determine if any significant bleeding or possible obstruction exists, which has not been noted on this case. Patient had minimal bleeding which cleared on subsequent suctioning. Her saturation was maintained normal above $90 \%$ and had stable vital signs, oriented and communicating well. There were no further complications, and control chest radiography revealed correct positioning of the tracheostomy tube.

Patient required longer intensive care management and was successfully weaned after eight weeks from ventilatory support. Patient discharged from ICU on 09-09-2008 with no identified neurological sequele. Three months after her discharge patient re-evaluated again in our follow up clinic and control chest radiography demonstrated full recovery with no residual pulmonary complications.

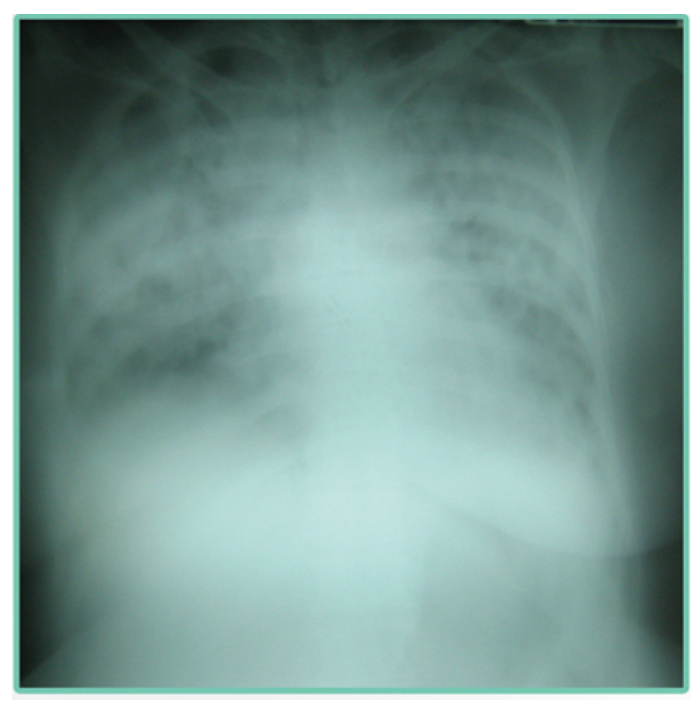

Chest radiography, diffuse bilateral infiltrates and signs of congestion.

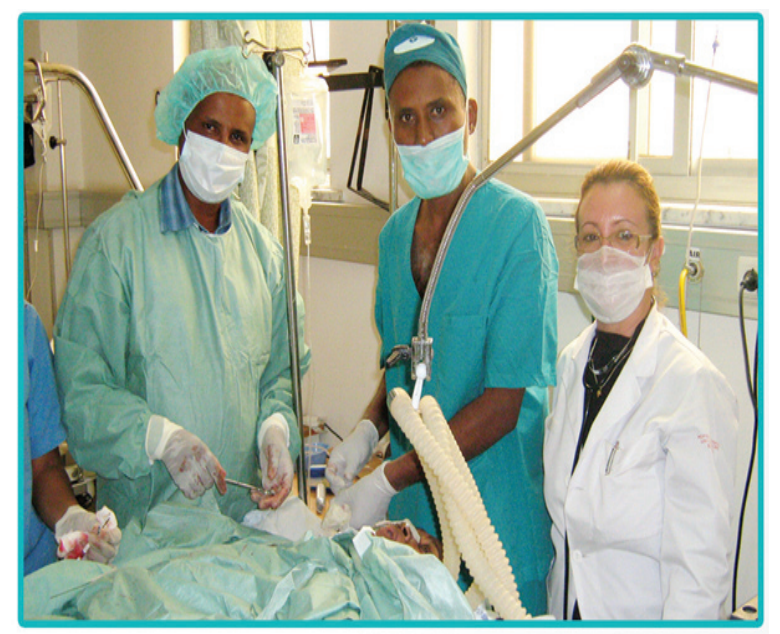

A bed side procedure in the intensive care unit.

\section{Discussion}

PDT is an established and safe intervention for tracheal access in intensive care. Low incidence of complications are reported, and the technique has gained widespread popularity. Besides PDT has several advantages when compared to oral endotracheal intubation: improves pulmonary hygiene and patient comfort, as well as facilitating mechanical weaning. In addition PDT is less expensive than open tracheostomy and avoids associated risks with transportation to operating suite in un stable patient.

In our case the procedure was successful and patient had no complications in her subsequent followups. How ever, further experience with this procedure is necessary to evaluate its over all efficacy and long term results.

\section{Acknowledgements}

The authors wish to thank Emergency Intensive Care and Anaesthesia Careggi Teaching University Hospital Firenze, for providing us continuous material support and training. In particular we wish to thank Dr Mebrahtu G/Hiwot, Dr Tesfagaber Halie and Mr G/Hiwot T.Bulla, for their in put in the management of this patient. The role played by our anaesthetist, Mr Teodros H/Mariam was remarkable and we are grateful. Last but not list we thank all our ICU staff for their dedication and the tremendous efforts in saving the life of our patients.

\section{References}

1 Elective PDT a new simple bed side procedure. Chest 1985; 87:715-9

2 Prospective comparison PDT with surgical tracheostomy. Intensive care Med 1991; 14:861-3

3 Management of the chronically ventilated patient with tracheostomy. Chronic respirator diseases $2005 ;$; (3):151-161

4. PDT for Emergency air way access: J intensive care med $2003 ;$; 4):222-226

5. Clinical review: PDT.Crit Care 2005; 10:202

6. Prevalence of tracheostomy in ICU patients. Intensive care med 2000;29:1390-3

7 PDT long term out come. Intensive Care Med ;1998;24:685 